\title{
On the Well Posedness of Vanishing Viscosity Limits
}

\author{
Alberto Bressan
}

\begin{abstract}
This paper provides a survey of recent results concerning the stability and convergence of viscous approximations, for a strictly hyperbolic system of conservation laws in one space dimension. In the case of initial data with small total variation, the vanishing viscosity limit is well defined. It yields the unique entropy weak solution to the corresponding hyperbolic system.
\end{abstract}

\section{Introduction}

Consider the Cauchy problem for a quasilinear system in one space dimension:

$$
u_{t}+A(u) u_{x}=0, \quad u(0, x)=\bar{u}(x) .
$$

We assume that the system is strictly hyperbolic, so that each $n \times n$ matrix $A(u)$ has real distinct eigenvalues

$$
\lambda_{1}(u)<\lambda_{2}(u)<\cdots<\lambda_{n}(u)
$$

and a basis of eigenvectors $r_{1}(u), \ldots, r_{n}(u)$ with unit length. Due to the nonlinearity of the equations, even for smooth initial data it is well known that the solution can lose regularity within finite time. Therefore, to obtain global existence of solutions, one has to work in a space of discontinuous functions and interpret the equation (1) in some relaxed sense.

In the conservative case where $A(u)=D f(u)$ is the Jacobian matrix of some flux function $f$, one can look for a weak solution of the system of conservation laws

$$
u_{t}+f(u)_{x}=0, \quad u(0, x)=\bar{u}(x) .
$$

More generally, one can consider the limit of solutions to the parabolic system

$$
u_{t}^{\varepsilon}+A\left(u^{\varepsilon}\right) u_{x}^{\varepsilon}=\varepsilon u_{x x}, \quad u^{\varepsilon}(0, x)=\bar{u}(x),
$$


letting the viscosity coefficient $\varepsilon \rightarrow 0$. A natural conjecture is that this limit exists, depends continuously on the initial data $\bar{u}$, and coincides with the entropy weak solution of (2) whenever the system is in conservation form. In the scalar case, this result has been known for a long time [O], also in several space dimensions [K]. In the case of systems of conservation laws, partial results in this direction were obtained by singular perturbations [GX] or compensated compactness [DP].

We consider here the case of general solutions of $n \times n$ systems, possibly not in conservation form. For initial data with small total variation, the stability and convergence of vanishing viscosity limits was recently proved in [BB]. On the other hand, for initial data $\bar{u} \in \mathbf{L}^{\infty}$, a counterexample given in [BS] shows the possible non-uniqueness of vanishing viscosity limits.

If the initial data has bounded but possibly large total variation, an example of Jenssen [J] shows that the solution of the system of conservation laws (2) can blow up in finite time. In such cases, it is expected (but not yet proved) that the vanishing viscosity limit is well posed only on an initial time interval, as long as the total variation remains bounded.

In the present note we shall outline the main ideas in the estimates [BB] on viscous approximations, and discuss the example in [BS]. For all details we refer to the original papers. In connection with the Cauchy problem for hyperbolic systems of conservation laws, a comprehensive presentation of existence, uniqueness and stability results can be found in [B3].

\section{Small BV data}

For strictly hyperbolic systems and initial data with small total variation, the following general well posedness result on vanishing viscosity limits was proved in [BB].

Theorem 1 Consider the Cauchy problem for the hyperbolic system with viscosity

$$
u_{t}^{\varepsilon}+A\left(u^{\varepsilon}\right) u_{x}^{\varepsilon}=\varepsilon u_{x x}^{\varepsilon}, \quad u^{\varepsilon}(0, x)=\bar{u}(x) .
$$

Assume that the matrices $A(u)$ are strictly hyperbolic, smoothly depending on $u$ in a neighborhood of a given state $u^{*} \in \mathbb{R}^{n}$. Then there exist constants $C, L$ and $\delta>0$ such that the following holds. If

$$
\text { Tot. Var. }\{\bar{u}\}<\delta, \quad \text { and } \quad\left|u^{*}-\lim _{x \rightarrow-\infty} \bar{u}(x)\right|<\delta,
$$

then for each $\varepsilon>0$ the Cauchy problem (4) has a unique solution $u^{\varepsilon}$, defined for all $t \geq 0$. Adopting a semigroup notation, this will be written as $t \mapsto u^{\varepsilon}(t, \cdot) \doteq S_{t}^{\varepsilon} \bar{u}$. In addition, one has the following uniform bounds on the total variation and Lipschitz stability estimates:

$$
\begin{aligned}
& \text { Tot. Var. }\left\{S_{t}^{\varepsilon} \bar{u}\right\} \leq C \text { Tot. Var. }\{\bar{u}\} \\
& \left\|S_{t}^{\varepsilon} \bar{u}-S_{t}^{\varepsilon} \bar{v}\right\|_{\mathbf{L}^{1}} \leq L\|\bar{u}-\bar{v}\|_{\mathbf{L}^{1}}
\end{aligned}
$$

As $\varepsilon \rightarrow 0+$, the solutions $u^{\varepsilon}$ converge to the trajectories of a continuous semigroup S such that

$$
\left\|S_{t} \bar{u}-S_{t} \bar{v}\right\|_{\mathbf{L}^{1}} \leq L\|\bar{u}-\bar{v}\|_{\mathbf{L}^{1}}
$$


These vanishing viscosity limits can be regarded as the unique vanishing viscosity solutions of the hyperbolic Cauchy problem

$$
u_{t}+A(u) u_{x}=0, \quad u(0, x)=\bar{u}(x) .
$$

In the conservative case $A(u)=D f(u)$, every vanishing viscosity solution is a weak solution of

$$
u_{t}+f(u)_{x}=0, \quad u(0, x)=\bar{u}(x),
$$

satisfying the Liu admissibility conditions [L].

In the remainder of this section, we shall outline the main ideas involved in the proof.

As a preliminary, observe that a function $u^{\varepsilon}$ is a solution of (4) if and only if

$$
u^{\varepsilon}(t, x)=u(t / \varepsilon, x / \varepsilon),
$$

where $u$ is a solution of

$$
u_{t}+A(u) u_{x}=u_{x x}
$$

with initial data $u(0, x)=\bar{u}(\varepsilon x)$. Clearly, the total variation is not affected by this rescaling. It thus suffices to prove uniform BV bounds on a solution to the parabolic problem (11) with unit viscosity. Our basic approach is the following.

(i) At each point $(t, x)$ the gradient $u_{x}$ is decomposed along a suitable basis of unit vectors, say

$$
u_{x}=\sum v_{i} \tilde{r}_{i}
$$

(ii) From (11) we then derive an equation describing the evolution of these gradient components

$$
v_{i, t}+\left(\tilde{\lambda}_{i} v_{i}\right)_{x}-v_{i, x x}=\phi_{i}
$$

(iii) Since the left hand side of (13) is in conservation form, by showing that all source terms $\phi_{i}=\phi_{i}(t, x)$ are integrable, we can conclude

$$
\left\|v_{i}(t, \cdot)\right\|_{\mathbf{L}^{1}} \leq\left\|v_{i}(0, \cdot)\right\|_{\mathbf{L}^{1}}+\int_{0}^{\infty} \int_{\mathbb{R}}\left|\phi_{i}(s, x)\right| \mathrm{d} x \mathrm{~d} s<\infty
$$

for all $t>0$. In turn, this implies

$$
\left\|u_{x}(t, \cdot)\right\|_{\mathbf{L}^{1}} \leq \sum\left\|v_{i}(t, \cdot)\right\|_{\mathbf{L}^{1}}<\infty .
$$

The choice of the basis $\left\{\tilde{r}_{1}, \ldots, \tilde{r}_{n}\right\}$ is of fundamental importance. A decomposition of the gradient $u_{x}$ along the eigenvectors $r_{i}(u)$ of the hyperbolic matrix $A(u)$ would seem quite natural. However, it does NOT work. Indeed, in the case where the solution $u$ is a travelling shock profile, we would obtain source terms which are not identically zero. Hence they are certainly not integrable over the domain $t \in[0, \infty[$. 
An alternative approach, proposed by S. Bianchini, is to decompose $u_{x}$ as a sum of gradients of viscous travelling waves. By a viscous travelling $i$-wave we mean a solution having the form

$$
w(t, x)=U(x-\sigma t),
$$

where the speed $\sigma$ is close to the $i$-th eigenvalue $\lambda_{i}$ of the hyperbolic matrix $A$. Clearly, the function $U$ must provide a solution to the second order O.D.E.

$$
U^{\prime \prime}=(A(U)-\sigma) U^{\prime}
$$

The basic idea for the decomposition is as follows. At each point $(t, x)$, given $\left(u, u_{x}, u_{x x}\right)$, we seek travelling wave profiles $U_{1}, \ldots, U_{n}$ such that

$$
\begin{gathered}
U_{i}(x)=u(x) \quad i=1, \ldots, n \\
\sum_{i} U_{i}^{\prime}(x)=u_{x}(x), \quad \sum_{i} U_{i}^{\prime \prime}(x)=u_{x x}(x) .
\end{gathered}
$$

In general, the system of algebraic equations (17)-(18) is under-determined, i.e. it admits infinitely many solutions. Indeed, for a given state $u$, one can assign arbitrarily a speed $\sigma \approx \lambda_{i}(u)$ and a vector $\mathbf{v} \in \mathbb{R}^{n}$. Then the O.D.E. (16) can be uniquely solved with initial data

$$
U(x)=u(x), \quad U^{\prime}(x)=\mathbf{v} .
$$

As a result, for each $i=1, \ldots, n$, there exists a family of viscous travelling $i$-waves satisfying (17), depending on $n+1$ scalar parameters. In all, this gives us $n(n+1)$ free parameters, far more then the data $\left(u_{x}, u_{x x}\right) \in \mathbb{R}^{n+n}$ in (18). To single out a unique solution, the first thing we need to do is to select a special subfamily of viscous travelling waves depending on the right number of parameters to fit the data. This is achieved by means of the center manifold theorem.

We start by writing the second order O.D.E. in (16) as a first order system:

$$
\dot{u}=v, \quad \dot{v}=(A(u)-\sigma) v, \quad \dot{\sigma}=0 .
$$

Linearizing at a point $P^{*}=\left(u^{*}, 0, \lambda_{i}\left(u^{*}\right)\right)$ we obtain the linear system with constant coefficients

$$
\dot{u}=v, \quad \dot{v}=\left(A\left(u^{*}\right)-\lambda_{i}\left(u^{*}\right)\right) v, \quad \dot{\sigma}=0 .
$$

This system has a null space $\mathcal{N}_{i}$ of dimension $n+2$. By the center manifold theorem there exists a manifold $\mathcal{M}_{i}$, tangent to $\mathcal{N}_{i}$ at the point $P^{*}$, locally invariant for the flow of the nonlinear system (19). Instead of looking at all viscous travelling profiles, we can now restrict ourselves to only those profiles corresponding to a trajectory on the center manifold $\mathcal{M}_{i}$. Since this manifold is $(n+2)$-dimensional, through each state $u \in \mathbb{R}^{n}$ there is a 2-parameter family of such profiles, say $U_{i}\left(u, v_{i}, \sigma_{i}\right)$. Here $v_{i}$ denotes the scalar amplitude of the wave, and $\sigma_{i}$ is the speed. Calling $\tilde{r}_{i}=\tilde{r}_{i}\left(u, v_{i}, \sigma_{i}\right)$ the unit vector parallel to $U_{i}^{\prime}$, this profile $U_{i}$ is characterized by the equations

$$
U_{i}^{\prime \prime}=\left(A\left(U_{i}\right)-\sigma_{i}\right) U_{i}^{\prime}, \quad U_{i}(0)=u, \quad U_{i}^{\prime}(0)=v_{i} \tilde{r}_{i}
$$


In terms of these special travelling profiles lying on the center manifold, it can be shown that the decomposition (17)-(18) can be performed uniquely.

We stress the substantial differences between the two decompositions

$$
u_{x}=\sum_{i} v_{i} r_{i}(u), \quad u_{x}=\sum_{i} v_{i} \tilde{r}_{i}\left(u, v_{i}, \sigma_{i}\right)
$$

which are used in the hyperbolic and in the parabolic case, respectively.

(i) In the hyperbolic case, the unit vectors $r_{i}(u)$ are the eigenvectors of the matrix $A(u)$, hence they depend only on the state $u$. On the other hand, the viscous eigenvectors $\tilde{r}_{i}\left(u, v_{i}, \sigma_{i}\right)$ which are introduced in (20) in terms of a center manifold, depend not only on $u$ but also on two additional scalar parameters: the amplitude $v_{i}$ and the speed $\sigma_{i}$ of a viscous travelling profile through $u$. These can be determined implicitly as functions of $u_{x}, u_{x x}$.

(ii) For $i=1, \ldots, n$, in addition to the eigenvalue $\lambda_{i}$ of the matrix $A(u)$, we can define the viscous eigenvalue $\tilde{\lambda}_{i}\left(u, v_{i}, \sigma_{i}\right)$ by setting

$$
\tilde{\lambda}_{i} \doteq\left\langle\tilde{r}_{i}, A(u) \tilde{r}_{i}\right\rangle
$$

Instead of the standard relation

$$
\left(A(u)-\lambda_{i}\right) r_{i}=0
$$

one can show that these viscous eigenvectors satisfy

$$
\left(A(u)-\tilde{\lambda}_{i}\right) \tilde{r}_{i}=v_{i}\left[\frac{\partial \tilde{r}_{i}}{\partial u} \tilde{r}_{i}+\left(\tilde{\lambda}_{i}-\sigma_{i}\right) \frac{\partial \tilde{r}_{i}}{\partial v_{i}}\right] .
$$

The presence of non-zero terms on the right hand side of (22) is of fundamental importance. Indeed, in the evolution equation (13) for the component $v_{i}$, these terms achieve a crucial cancellation with other terms that would otherwise not be integrable.

By differentiating (11), we obtain a system of evolution equations for the components $v_{i}$, of the form (13). To achieve a proof of the BV estimates, one needs to show that the source terms $\phi_{i}=\phi_{i}(t, x)$ on the right hand side of (13) are integrable on the domain $\{t>0, x \in \mathbb{R}\}$. The key idea is that these source terms are due to different types of wave interactions. They can be controlled by suitable interaction functionals, similar to the one introduced by Glimm [G] in connection with hyperbolic systems. We describe below the three basic ones. Consider first two independent, scalar diffusion equations with strictly different drifts:

$$
\left\{\begin{array}{r}
z_{t}+[\lambda(t, x) z]_{x}-z_{x x}=0 \\
z_{t}^{*}+\left[\lambda^{*}(t, x) z^{*}\right]_{x}-z_{x x}^{*}=0
\end{array}\right.
$$

assuming that

$$
\inf _{t, x} \lambda^{*}(t, x)-\sup _{t, x} \lambda(t, x) \geq c>0 .
$$


We regard $z$ as the density of waves with a slow speed $\lambda$ and $z^{*}$ as the density of waves with a fast speed $\lambda^{*}$. A transversal interaction potential is defined as

$$
\begin{gathered}
Q\left(z, z^{*}\right) \doteq \frac{1}{c} \iint_{\mathbb{R}^{2}} K\left(x_{2}-x_{1}\right)\left|z\left(x_{1}\right)\right|\left|z^{*}\left(x_{2}\right)\right| \mathrm{d} x_{1} \mathrm{~d} x_{2}, \\
K(y) \doteq \begin{cases}e^{-c y / 2} & \text { if } y>0, \\
1 & \text { if } y \leq 0 .\end{cases}
\end{gathered}
$$

One can show that this functional $Q$ is monotonically decreasing along every couple of solutions $z, z^{*}$. The total amount of interaction between fast and slow waves can now be estimated as

$$
\begin{aligned}
\int_{0}^{\infty} \int_{\mathbb{R}}|z(t, x)|\left|z^{*}(t, x)\right| \mathrm{d} x \mathrm{~d} t & \leq-\int_{0}^{\infty}\left[\frac{d}{d t} Q\left(z(t), z^{*}(t)\right)\right] \mathrm{d} t \\
& \leq Q\left(z(0), z^{*}(0)\right) \leq \frac{1}{c} \int_{\mathbb{R}}|z(0, x)| \mathrm{d} x \cdot \int_{\mathbb{R}}\left|z^{*}(0, x)\right| \mathrm{d} x .
\end{aligned}
$$

By means of Lyapunov functionals of this type we can control all source terms due to the interaction of waves of different families.

Next, to control the interactions between waves of the same family, we seek functionals which are decreasing along every solution of the scalar viscous conservation law

$$
u_{t}+f(u)_{x}=u_{x x} .
$$

For this purpose, to a scalar function $x \mapsto u(x)$ we associate the curve in the plane

$$
\gamma \doteq\left(\begin{array}{c}
u \\
f(u)-u_{x}
\end{array}\right)=\left(\begin{array}{c}
\text { conserved quantity } \\
\text { flux }
\end{array}\right)
$$

In connection with a solution $u=u(t, x)$ of $(23)$, the curve $\gamma$ evolves according to

$$
\gamma_{t}+f^{\prime}(u) \gamma_{x}=\gamma_{x x}
$$

Notice that the vector $f^{\prime}(u) \gamma_{x}$ is parallel to $\gamma$, hence the presence of this term in (25) only amounts to a reparametrization of the curve, and does not affect its shape. The curve thus evolves in the direction of curvature. An obvious Lyapunov functional is the length of the curve. In terms of the variables

$$
\gamma_{x}=\left(\begin{array}{c}
v \\
w
\end{array}\right) \doteq\left(\begin{array}{c}
u_{x} \\
-u_{t}
\end{array}\right)
$$

this length is given by

$$
L(\gamma) \doteq \int\left|\gamma_{x}\right| \mathrm{d} x=\int \sqrt{v^{2}+w^{2}} \mathrm{~d} x
$$

We can estimate the rate of decrease in the length as

$$
-\frac{d}{d t} L(\gamma(t))=\int_{\mathbb{R}} \frac{|v|\left[(w / v)_{x}\right]^{2}}{\left(1+(w / v)^{2}\right)^{3 / 2}} \mathrm{~d} x \geq \frac{1}{\left(1+\delta^{2}\right)^{3 / 2}} \int_{|w / v| \leq \delta}|v|\left[(w / v)_{x}\right]^{2} \mathrm{~d} x,
$$


for any given constant $\delta>0$. This yields a useful a priori estimate on the integral on the right hand side of (28):

$$
\int_{0}^{\infty} \int_{|w / v| \leq \delta}|v|\left[(w / v)_{x}\right]^{2} \mathrm{~d} x \mathrm{~d} t=\mathcal{O}(1) \cdot \int_{0}^{\infty}\left|\frac{d}{d t} L(\gamma(t))\right| \mathrm{d} t=\mathcal{O}(1) \cdot L(\gamma(0)) .
$$

In connection with the same curve $\gamma$ in (24), we now introduce a second functional, defined in terms of a wedge product.

$$
Q(\gamma) \doteq \frac{1}{2} \iint_{x<x^{\prime}}\left|\gamma_{x}(x) \wedge \gamma_{x}\left(x^{\prime}\right)\right| \mathrm{d} x \mathrm{~d} x^{\prime}
$$

For any curve that moves in the plane in the direction of curvature, one can show that this functional is monotone decreasing and its decrease bounds the area swept by the curve: $|d A| \leq-d Q$.

Using (25)-(26) we now compute

$$
-\frac{d Q}{d t} \geq\left|\frac{d A}{d t}\right|=\int\left|\gamma_{t} \wedge \gamma_{x}\right| \mathrm{d} x=\int\left|\gamma_{x x} \wedge \gamma_{x}\right| \mathrm{d} x=\int\left|v_{x} w-v w_{x}\right| \mathrm{d} x .
$$

Integrating w.r.t. time, we thus obtain onother useful a priori bound:

$$
\int_{0}^{\infty} \int\left|v_{x} w-v w_{x}\right| \mathrm{d} x \mathrm{~d} t \leq \int_{0}^{\infty}\left|\frac{d Q(\gamma(t))}{d t}\right| \mathrm{d} t \leq Q(\gamma(0)) .
$$

Together, the functionals in (27) and (29) allow us to estimate all source terms in (13) due to the interaction of waves of the same family.

This yields the $\mathbf{L}^{1}$ estimates on the source terms $\phi_{i}$, in (29), proving the uniform bounds on the total variation of a solution $u$ of (11). See [BB] for details.

To prove the uniform stability of all solutions of the parabolic system (11) having small total variation, we consider the linearized system describing the evolution of a first order variation. Inserting the formal expansion $u=u_{0}+\epsilon z+O\left(\epsilon^{2}\right)$ in (11), we obtain

$$
z_{t}+[D A(u) \cdot z] u_{x}+A(u) z_{x}=z_{x x} .
$$

Our basic goal is to prove the bound

$$
\|z(t)\|_{\mathbf{L}^{1}} \leq L\|z(0)\|_{\mathbf{L}^{1}},
$$

for some constant $L$ and all $t \geq 0$ and every solution $z$ of (30). By a standard homotopy argument, from (31) one easily deduces the Lipschitz continuity of the solution of (11) on the initial data. Namely, for every couple of solutions $u, \tilde{u}$ with small total variation one has

$$
\|u(t)-\tilde{u}(t)\|_{\mathbf{L}^{1}} \leq L\|u(0)-\tilde{u}(0)\|_{\mathbf{L}^{1}} .
$$

To prove (31) we decompose the vector $z$ as a sum of scalar components: $z=\sum_{i} h_{i} \tilde{r}_{i}$, write an evolution equation for these components:

$$
h_{i, t}+\left(\tilde{\lambda}_{i} h_{i}\right)_{x}-h_{i, x x}=\hat{\phi}_{i}
$$


and show that the source terms $\hat{\phi}_{i}$ are integrable on the domain $\{t>0, x \in \mathbb{R}\}$. For all details we always refer to $[\mathrm{BB}]$.

For every initial data $u(0, \cdot)=\bar{u}$ with small total variation, the previous arguments yield the existence of a unique global solution to the parabolic system (11), depending Lipschitz continuously on the initial data, in the $\mathbf{L}^{1}$ norm. Performing the rescaling $t \mapsto t / \varepsilon, x \mapsto x / \varepsilon$, we immediately obtain the same results for the Cauchy problem (4). Adopting a semigroup notation, this solution can be written as $u^{\varepsilon}(t, \cdot)=S_{t}^{\varepsilon} \bar{u}$. Thanks to the uniform bounds on the total variation, a compactness argument yields the existence of a strong limit in $\mathbf{L}_{\text {loc }}^{1}$

$$
u=\lim _{\varepsilon_{m} \rightarrow 0} u^{\varepsilon_{m}}
$$

at least for some subsequence $\varepsilon_{m} \rightarrow 0$. Since the $u^{\varepsilon}$ depend continuously on the initial data, with a uniform Lipschitz constant, the same is true of the limit solution $u(t, \cdot)=S_{t} \bar{u}$. In the conservative case where $A(u)=D f(u)$, it is not difficult to show that this limit $u$ actually provides a weak solution to the Cauchy problem (2).

The only remaining issue is to show that the limit in (33) is unique, i.e. it does not depend on the subsequence $\left\{\varepsilon_{m}\right\}$. In the standard conservative case, this fact can already be deduced from the uniqueness result in $[B G]$. In the general case, uniqueness is proved in two steps. First we show that, in the special case of a Riemann problem, the solution obtained as vanishing viscosity limit is unique and can be completely characterized. To conclude the proof, we then rely on the same general argument as in [B2]: if two Lipschitz semigroups $S, S^{\prime}$ provide the same solutions to all Riemann problems, then they must coincide.

\section{Initial data with unbounded variation}

We discuss here an example, first appeared in [BS], showing that the vanishing viscosity limit may not be unique, for strictly hyperbolic systems with $\mathbf{L}^{\infty}$ data. Consider the $3 \times 3$ system

$$
u_{t}+u_{x}=0, \quad v_{t}-v_{x}=0, \quad w_{t}+\frac{1-u v}{6} \cdot w_{x}=0,
$$

with initial data having unbounded variation:

$$
\begin{gathered}
u(0, x)=v(0, x)=\varphi(x) \doteq \begin{cases}1 & \text { if } 2^{-2 n-1}<|x|<2^{-2 n} \\
-1 & \text { if } 2^{-2 n}<|x|<2^{-2 n+1}\end{cases} \\
w(0, x)= \begin{cases}0 & \text { if } x<0 \\
1 & \text { if } x>0 .\end{cases}
\end{gathered}
$$

Observe that the system is strictly hyperbolic as long as $u, v \in[-1,1]$. Indeed, in this case the characteristic speeds are

$$
\lambda_{1}=-1, \quad \lambda_{2}=\frac{1-u v}{6} \in[0,1 / 3], \quad \text { and } \quad \lambda_{3}=1 .
$$




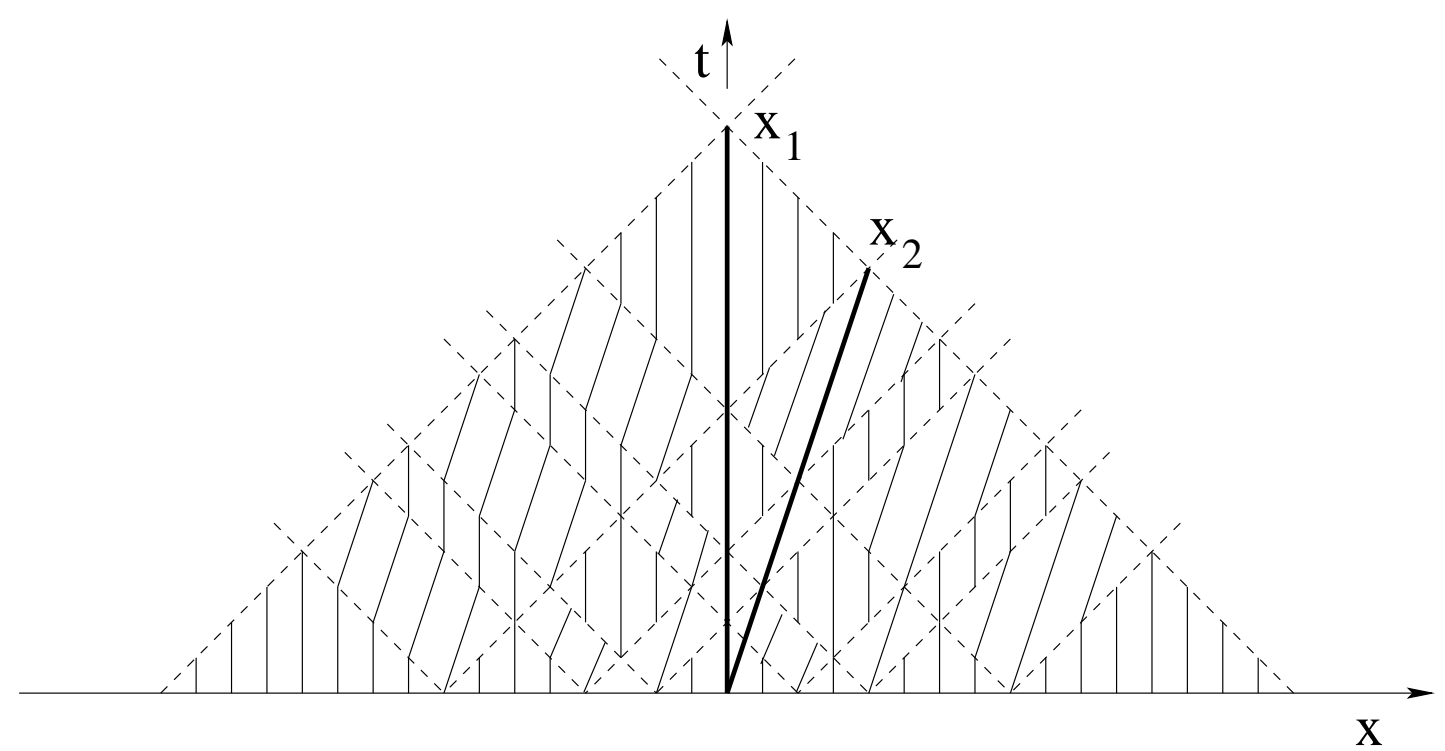

Figure 1: multiple solutions.

Of course, the first two components of the solution are given by

$$
u(t, x)=\varphi(x-t), \quad v(t, x)=\varphi(x+t) .
$$

The non-uniqueness stems from the fact that the O.D.E. for characteristics of the second family

$$
\dot{x}=\frac{1-u v}{6}
$$

has a discontinuous right hand side. By strict hyperbolicity, these discontinuities are transversal to all trajectories of (35). If the total variation of $u, v$ were bounded, one could apply the uniqueness theorem in [B1] and deduce the uniqueness and Lipschitz continuous dependence of solutions to this O.D.E.. In the present case, however, the total variation of $u, v$ is infinite, and multiple solutions appear. In particular (fig. 1), the trajectories $x_{1}(t)=0$ and $x_{2}(t)=t / 3$ are both Caratheodory solutions. Since all three characteristic fields are linearly degenerate, the two solutions

$$
w_{1}(t, x)=\left\{\begin{array}{ll}
0 & \text { if } x<0, \\
1 & \text { if } x>0,
\end{array} \quad w_{2}(t, x)= \begin{cases}0 & \text { if } x<t / 3, \\
1 & \text { if } x>t / 3\end{cases}\right.
$$

can both be obtained as limits of smooth solutions. Therefore, they can also be obtained as limits of viscous approximations.

\section{References}

[BB] S. Bianchini and A. Bressan, Vanishing viscosity solutions to nonlinear hyperbolic systems, Preprint S.I.S.S.A., Trieste 2001.

[B1] A.Bressan, Unique solutions for a class of discontinuous differential equations, Proc. Amer. Math. Soc. 104 (1988), 772-778. 
[B2] A. Bressan, The unique limit of the Glimm scheme, Arch. Rational Mech. Anal. 130 (1995), 205-230.

[B3] A. Bressan, Hyperbolic Systems of Conservation Laws. The One Dimensional Cauchy Problem. Oxford University Press, 2000.

[BG] A. Bressan and P. Goatin, Oleinik type estimates and uniqueness for $n \times n$ conservation laws, J. Diff. Equat. 156 (1999), 26-49.

[BLY] A. Bressan, T. P. Liu and T. Yang, $L^{1}$ stability estimates for $n \times n$ conservation laws, Arch. Rational Mech. Anal. 149 (1999), 1-22.

[BS] A. Bressan and W. Shen, Uniqueness for discontinuous O.D.E. and conservation laws, Nonlinear Analysis, T. M. A. 34 (1998), 637-652.

[DP] R. DiPerna, Convergence of approximate solutions to conservation laws, Arch. Rational Mech. Anal. 82 (1983), 27-70.

[G] J. Glimm, Solutions in the large for nonlinear hyperbolic systems of equations, Comm. Pure Appl. Math. 18 (1965), 697-715.

[GX] J. Goodman and Z. Xin, Viscous limits for piecewise smooth solutions to systems of conservation laws, Arch. Rational Mech. Anal. 121 (1992), 235265.

[K] S. Kruzhkov, First order quasilinear equations with several space variables, Math. USSR Sbornik 10 (1970), 217-243.

[J] H. K. Jenssen, Blowup for systems of conservation laws, SIAM J. Math. Anal. 31 (2000), 894-908.

[L] T. P. Liu, Admissible solutions of hyperbolic conservation laws, Amer. Math. Soc. Memoir 240 (1981).

[O] O. Oleinik, Discontinuous solutions of nonlinear differential equations (1957), Amer. Math. Soc. Translations 26, 95-172.

[V] A. Vanderbauwhede, Centre manifolds, normal forms and elementary bifurcations, Dynamics Reported, Vol. 2 (1989), 89-169.

S.I.S.S.A., Via Beirut 4, Trieste 34014 Italy.

bressan@sissa.it 